\title{
Load Adaptive Networking \& Energy Aware System Design to Reduce the Energy Consumption in Telecommunication Networks
}

\author{
Mohd shoebuddin mujeeb \\ Jawahar lal Nehru Technological University \\ Vardhaman College Of Engineering \\ Shamshabad, Hyderabad, India.
}

\author{
S.Srinivas \\ Jawahar Lal Nehru Technological University \\ Vardhaman College of Engineering \\ Shamshabad, Hyderabad, India.
}

\begin{abstract}
Worldwide, the growth rate of Internet users is about 20 percent per year. In developing countries this growth rate is closer to $40-50$ percent. One of the main challenges for the future of information and communication technologies is reduction of the power consumption in telecommunication networks.

We introduce the different network architectures and the design parameters that define their power consumption. Based on these parameters the power consumption is then quantified. We elaborate on approaches for the reduction of power consumption and best communication
\end{abstract}

Keywords: Communication, Internet, Power consumption.

\section{INTRODUCTION}

DSL

DSL stands for Digital Subscriber Line. It is a family of technologies that provide Internet access by transmitting digital data over the wires of a local telephone network. In telecommunications marketing, bit rate of consumer DSL services typically ranges from $256 \mathrm{kbit} / \mathrm{s}$ to $40 \mathrm{Mbit} / \mathrm{s}$ downstream, depending on DSL technology, line conditions.

DSL is more like the older 'modems' in that they do use the standard copper phone lines that are in your house. It requires that there be a special switch installed at your local phone company's main routing station but DSL also has a limitation of a 4 mile. The cool feature of DSL is that you do not need to have a 'special' line installed.

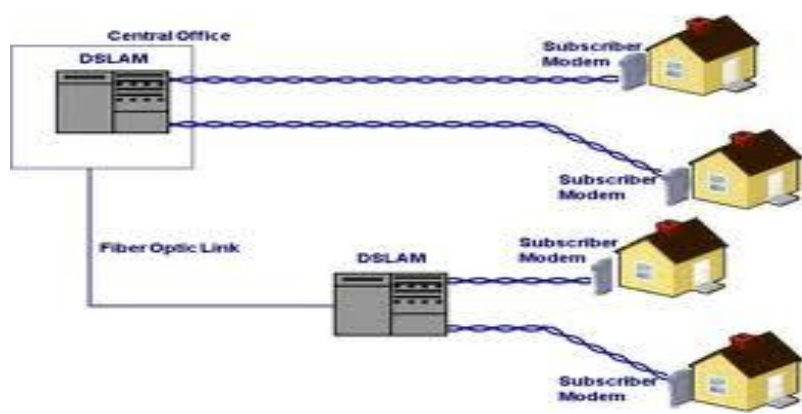

Fig. shows the Dsl System
DSL or SDSL offers the most bandwidth but they are more expensive. In SDSL S stands for synchronous or Symmetric, this means that the bandwidth of both the upstream and the downstream are the same speed. Depending on the package you sign up for and how far away from the switch you are, the actual speed you get can vary. The most common speeds are 256k (256kilobits)/second and $1 \mathrm{Mb}$ (1megabit)/second. Asynchronous Digital Subscriber Line (ADSL) is same as the SDSL, but the upstream bandwidth is smaller than the downstream. Common ADSL speeds are $256 \mathrm{~kb}$ downstream - 96kb upstream, $1 \mathrm{mb}$ downstream $256 \mathrm{~kb}$ upstream.

Peer-to-peer is a communications model in which each party has the same capabilities and either party can initiate a communication session. It will be contrasted with other models which include the master/slave model and the client/server model. In some cases, peer-to-peer communications is implemented by giving each communication node both server and client capabilities

ADSL is a type of DSL where the upstream and downstream speeds differ. There are many variations of DSL technology for different types of application (HDSL, SDSL, etc). 


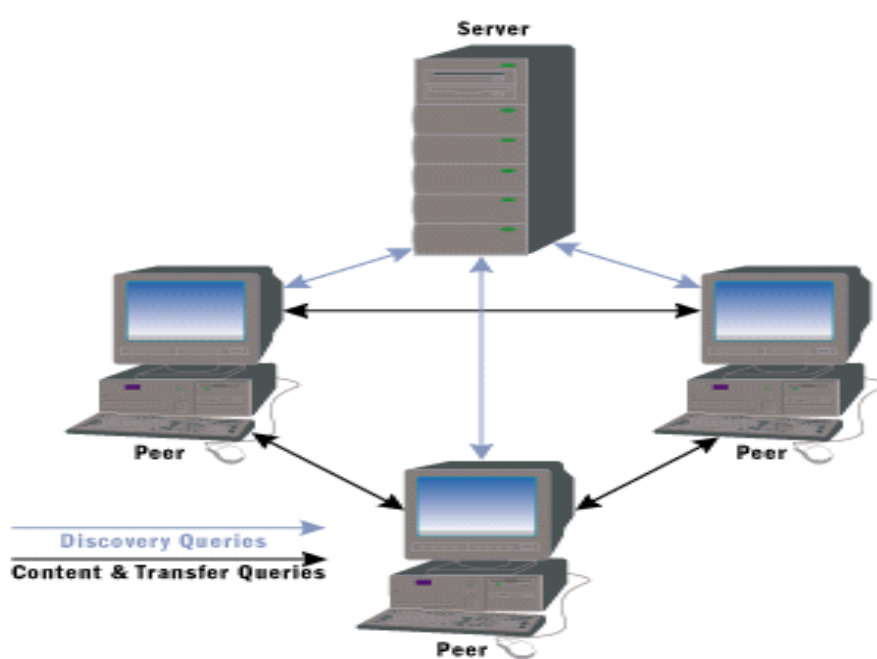

Fig. Peer to peer communication

\section{Related work:}

[1]In this paper, author demonstrated that the best opportunities for power savings come via best-of-breed technology, protocol simplification, and silicon and software optimization to achieve the least amount of processing necessary to move packets.

Pros and Cons:

--This approach is efficient under power savings.

--If a router is super-fast but lacks the required functionality and features needed to build a robust and secure communication infrastructure, it will find limited use.

[2] In this paper author proposed an anomaly detection scheme using dynamic training method in which the training data is updated at regular time intervals. Results show the effectiveness of our scheme compared with conventional scheme.

Pros and Cons:

--Presented a new detection method based on dynamically updated training data.

--Static training method could not be utilized efficiently.

[3]As Information and Communication Technology (ICT) is becoming more and more wide-spread and pervasive in our daily life, it is important to get a realistic overview of the worldwide impact of ICT on the environment in general and on energy and electricity needs in particular.

Pros and Cons:

--This mechanism decreased the power consumption.

--Power optimizations of the hardware of individual devices are not discussed.

[4] In this paper author proposed specific detection rules that can make legitimate nodes become aware of the threat, while the attack is still carrying on. Finally, the attack and present some implementation details that emphasize the little effort that an attacker would need to put in order to break into a realistic sensor network.

Pros and Cons:

--They opened the way for defining more general and formal rules in intrusion detection systems.

--Energy Efficiency of sensors is not so clarified.

[5] In this paper author examined the somewhat controversial subject motivated by data collected by the U.S. Department of Commerce i.e., energy consumption of networking devices in the Internet.

Pros and Cons:

--Energy is a precious resource whose scarcity hinders widespread Internet deployment particularly in the developing world. They showed that the impact of saving energy is huge.

--Maximization of the amount of energy conservation is not shown.

\section{METHODOLOGY:}

\section{Existing system:}

In existing technology, energy saving is done by putting network interfaces and other router \& switch components to sleep. However, currently new approaches or variations on the suggested approaches are emerging and also the share of the core networks could become significant as well with increasing bit rates.

Is it possible to connect from one DSL modem to another (eg., from house to house)?

Yes you can connect 2 DSL users together using a VPN to make a WAN, only requirement is a router on both sides that can do the IPSec, PPTP, etc.

From your house to the $\mathrm{CO}$, distance only matters for DSL. If you create a VPN then you will go from your house to the CO's router then to your friend's house but not be going directly from your house to whoever else's house, the distance only figures into if you can get DSL and at what speeds, it won't matter for VPNs.

\section{Proposed model:}

In this analysis we focus on the optical and DSL technologies. We elaborate on approaches for the reduction of power consumption. At present, in core networks the power consumption is relatively low.

Is it possible to connect from one DSL modem to another (eg., from house to house) directly?

Yes you can connect 2 DSL users together to make a communication, only requirement is a router on both sides 
In our proposed model, to improve power saving there is no need of $\mathrm{CO}$ for providing communication, but $\mathrm{CO}$ is needed only for selecting best peer, communication will be done through the intermediate routers and switches...

\section{MODULES:}

We are dividing our project work into small modules, they are given as bellow

- Network Design

- Wireless Network Design

- Load Balancing.

To make our project as efficient we introduced one more model in our work

$$
\text { - Best peer selection }
$$

\subsection{Network Design:}

Network design and planning is an iterative process, encompassing topological design and network-realization.

a. Topological design: This stage involves determining where to place the components and how to connect them. The (topological) optimization methods

b. Network realization: This stage involves determining how to meet capacity requirements and ensure reliability within the network. This involves determining all information relating to demand and reliability and then using this information to calculate an actual physical circuit plan.

\subsection{Wireless Network Design:}

The energy efficiency of wireless access networks can be improved by increasing the ranges of the base stations. Thus, larger areas can be covered by a single base station, and fewer base stations are necessary. This can be done by the use of multiple transmitting and receiving antennas. This technique is known as multiple-input multiple-output.

For example, 2 transmitting and 2 receiving antennas, the range increases by 66 percent, while the power consumption increases only by 2 to 4 percent, resulting in higher energy efficiency.

\subsection{Load Balancing:}

Many energy aware routing algorithms and protocols have been proposed for networks recently to achieve aims like minimum energy consumption, maximized network lifetime, overhead and reduced communication latency. Load balancing is a networking method to distribute workload across multiple clusters, network links to achieve optimal resource utilization, minimize response time, maximize throughput and avoid overload.

In our proposed model, we made the model with peer to peer technology. In this model, we are using the centralized device name as server (or service provider or $\mathrm{CO}$ ) for searching the devices, which satisfying the needs. After searching the devices we are going to make the communication through the intermediate switches without $\mathrm{CO}$.

In this module, to improve load balancing we are using the two type of requesting option.

- Unicast type

- Multicast type

Unicast

- Mainly used in initial query to CO (it's making the problem in multiple user searches, so in that operation we are making multicasting).

1 Multicast

- Query from CO to peers (mostly multicast is used in data transmission to multiple users, but in our system we are proposing to make request).

\subsection{Best Peer Selection:}

In this module, we are introducing the feedback function. By using feedback analyzing requester can select best peer for communication. In feedback service user can provide positive or negative feedback. If peer is satisfying the need then user will provide '+ve' otherwise '-ve'. More +ve value shows the peer is best one.

\section{RESULTS:}

In this paper, we have given the model testing analysis result.

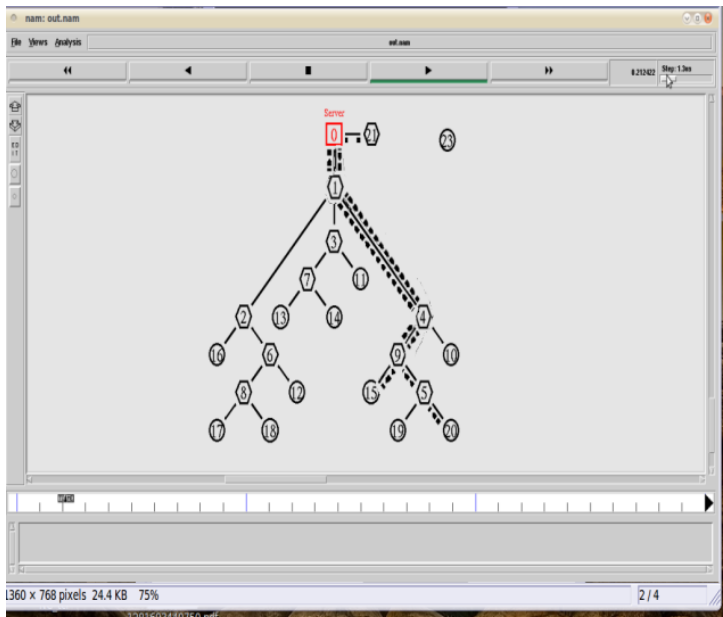

Fig. shows the model of previous system.

To improve data delivery and reduce energy consumption, we proposed a method with load balancing. 


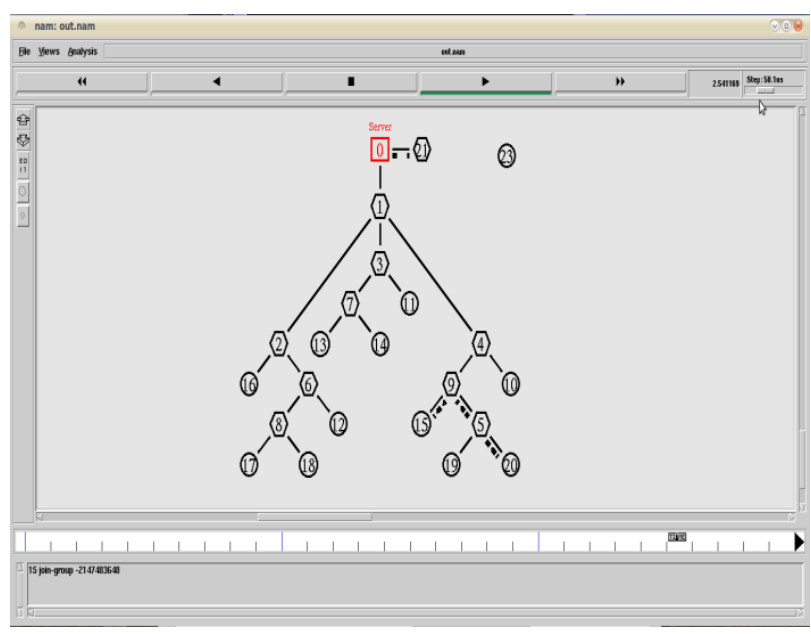

Fig. shows the model of Load Balancing system

From our analysis, we got the result with improved energy saving method. In this model we can save more energy compare than previous method. Analyzed result is given bellow.

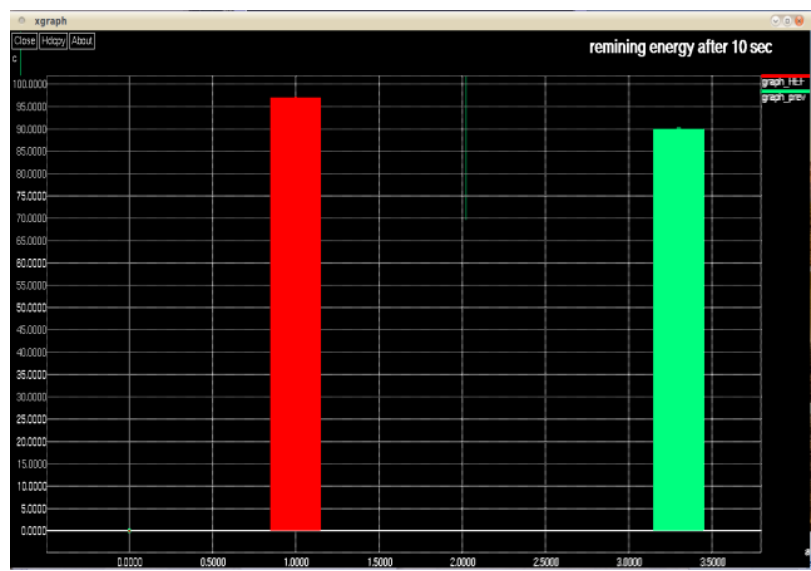

Fig. shows the Energy comparison between the Existing system and Proposed System.

First bar result is for previous model and second one is for proposed model. From this result we improved power saving upto $10 \%$. Here we analyzed with less number of intermediate switches. If there is more intermediate switches mean we can reduce $50 \%$ of power loss.

\section{CONCLUSION:}

By our proposed technique, we can improve the power saving as well as high quality network performance. And also we proposed feedback based security mechanism, communication. Here we developed theory based network simulation. In future, we will implement this in real time to make green city.

\section{ACKNOWLEDGEMENT:}

We wish to acknowledge the efforts of ECE Dept of Vardhaman College of Engineering for guidance which helped us work hard towards producing this research work.

\section{REFERENCES:}

[1] "Power saving strategies and technologies in network equipment opportunities and challenges, risk and rewards"., Luc Ceuppens, Alan Sardella, Daniel Kharitonov- 2009.

[2] "Detecting Blackhole Attack on AODV-based Mobile Ad Hoc Networks by Dynamic Learning Method"., Satoshi Kurosawa, Hidehisa Nakayama, Nei Kato, Abbas Jamalipour, and Yoshiaki Nemoto- 2005.

[3] "Worldwide Energy Needs for ICT: the Rise of PowerAware Networking"., mario pickavet, willem vereecken, sofie demeyer, pieter audenaert - 2008.

[4] "Launching a Sinkhole Attack in Wireless Sensor Networks; the Intruder Side"., Ioannis Krontiris, Thanassis Giannetsos, Tassos Dimitriou - 2008.

[5] "Greening of the Internet"., Maruti Gupta and Suresh Singh-2003.

[6] "Bandwidth Estimation: Metrics, Measurement Techniques, and Tools" Ravi Prasad, Constantinos Dovrolis, Margaret Murray and kc claffy - 2003.

[7] "Bandwidth Recycling in IEEE 802.16 Networks"., David Chuck and J. Morris Chang - 2010.

[8] "QoS-Oriented Intersystem Handover between IEEE 802.11b and Overlay Networks" Alexandre V. Garmonov, Seok Ho Cheon, Do Hyon Yim, Ki Tae Han, Yun Sang Park, Andrew Y. Savinkov, Stanislav A. Filin, Sergey N. Moiseev, and Mikhail S. Kondakov - 2008.

[9] "Power Consumption in Telecommunication networks: Overview and Reduction Strategies" Willem Vereecken, Ward Van Heddeghem, Margot Deruyck, Bart Puype, Bart Lannoo $-2011$.

[10] "Dynamic Source Routing in Ad Hoc Wireless Networks"., David B.Johnson, David A. Maltz - 1996. 